\title{
Shape deformations and angular momentum transfer in trapped Bose-Einstein condensates
}

\author{
F. Dalfovo ${ }^{1}$ and S. Stringari ${ }^{2}$ \\ ${ }^{1}$ Dipartimento di Matematica e Fisica, Università Cattolica, Via Musei 41, Brescia, Italy \\ and Istituto Nazionale per la Fisica della Materia, Unità di Brescia \\ ${ }^{2}$ Dipartimento di Fisica, Università di Trento, I-38050 Povo, Italy \\ and Istituto Nazionale per la Fisica della Materia, Unità di Trento
}

(September 13, 2000)

Angular momentum can be transferred to a trapped BoseEinstein condensate by distorting its shape with an external rotating field, provided the rotational frequency is larger than a critical frequency fixed by the energy and angular momentum of the excited states of the system. By using the GrossPitaevskii equation and sum rules, we explore the dependence of such a critical frequency on the multipolarity of the excitations and the asymmetry of the confining potential. We also discuss its possible relevance for vortex nucleation in rotating traps.

PACS numbers: 03.65.-w, 05.30.jp, 32.80.-t, 67.40.Db

It is well known that in order to create excitations in a superfluid by moving an impurity one has to overcome some critical velocity, which is fixed by the spectrum of the excited states of the system. In the case of a rectilinear motion of a heavy object, conservation of energy and momentum yields the critical velocity $v_{c}=\min [\epsilon(p) / p]$, where $\epsilon(p)$ is the energy of an excitations carrying momentum $p$; this is known as Landau criterion [1]. The external perturbation can also be a moving boundary, like the wall of a container, which produces excitations through its roughness. In liquid helium such a critical velocity has been the object of a longstanding investigation, involving the structure of the phonon-roton branch and the nucleation and dynamics of quantized vortex lines and rings. The existence of similar critical velocities in trapped Bose-Einstein condensates of alkali atoms is currently under study. In Ref. [2] an external potential (a laser beam) is used to produce a hole in the condensate and this hole is moved back and forth at variable velocity, playing the role of a massive impurity. Theoretical interpretations in terms of the Landau criterion have been already presented in Refs. [3, 1 . Here we consider the analog process in which the perturbation acts at the surface of the condensate, producing a rotating shape deformation. This kind of perturbation has been recently implemented in [5] to excite quadrupole and octopole modes, but the analysis is also relevant for rotating traps, like the one used in Ref. [6] to produce quantized vortices. The similarity with liquid helium is again worth stressing; in that case, when the liquid fills a rotating bucket, the roughness of the wall can transfer angu- lar momentum to the superfluid, favoring the occurrence of quantized vorticity above some critical angular velocity [7]. However, since the crucial length scales (healing length, surface thickness, interatomic distance, etc.) in liquid helium and trapped condensates are different, the role of surface excitations on the Landau criterion is also very different. In particular, surface modes can be ignored in the analysis of the experiments with helium in rotating vessels, while they might be crucial for gases of alkali atoms in rotating traps, as discussed below.

Let us consider a condensate which is initially in its ground state in an axially symmetric trap, with trapping frequencies $\omega_{\perp}$ and $\omega_{z}=\lambda \omega_{\perp}$, where $\lambda$ is the anisotropy parameter of the harmonic confining potential. Then, let us imagine a small distortion of the condensate caused by an external perturbation which rotates around the $z$ axis at angular velocity $\Omega$. This perturbation may be a modulation of the confining potential, a rotating laser beam, or something else; we only assume here that the consequent distortion of the condensate is indeed a shape deformation, coupled to the surface modes of the system (ripplon-like waves). Let us consider the process in which the external probe produces a single excited state, corresponding to a surface wave with energy $\hbar \omega(\ell)$ and angular momentum $\ell$ along $z$ (azimuthal quantum number $m=\ell$ ). One can assign a moment of inertia $I$ to the rotating perturbation and write the conservation laws for energy and angular momentum, letting $I \rightarrow \infty$ at the end. The simple result is that the conservation laws are both satisfied only if $\Omega$ is larger than the critical angular velocity $\Omega_{c}=\min [\omega(\ell) / \ell]$. This is the analog of the Landau criterion. If the rotation is slower than $\Omega_{c}$, the condensate simply adjusts its shape to the rotating perturbation by moving as an irrotational and nondissipative fluid. Conversely, if the rotation is faster than $\Omega_{c}$, the external perturbation can excite surface modes in a continuous way, transferring energy and angular momentum to the condensate.

The excitation spectrum of a condensate can be accurately evaluated by solving the linearized GrossPitaevskii (GP) equation, also known as Bogoliubov's equations [8]. The theory provides the energy of the excited states at zero temperature in terms of the relevant quantum numbers (number of radial nodes and angular momentum). For our purposes what matters is the 
energy $\hbar \omega(\ell)$ of the surface modes, i.e., the ones with no radial nodes. In a previous work, we already presented some results for the critical angular velocity $\Omega_{c}$ in a spherical condensate [9]. Here we discuss more systematically the consequences of this critical behavior and provide results for nonspherical traps.

We avoid the complication of solving the Bogoliubov's equations for axially symmetric condensates by using sum rules. As already shown in [10], the sum rule approach provides rigorous upper bounds to the dispersion $\omega(\ell)$ using the ground state density, solution of the stationary GP equation, as main ingredient. The key quantities are the $k$-energy weighted moments, $m_{k}=\int_{0}^{\infty} S_{F}(E) E^{k} d E$ of the dynamic structure factor, $S_{F}(E)=\sum_{j}|\langle j|F| 0\rangle|^{2} \delta\left(E-E_{j 0}\right)$, associated with a given excitation operator $F$, where $E_{j 0}=\left(E_{j}-E_{0}\right)$ is the excitation energy of the eigenstate $|j\rangle$ of the Hamiltonian. The $m_{1}$ and $m_{3}$ moments can be written as expectation values of commutators, $m_{1}=(1 / 2)\left\langle 0\left|\left[F^{\dagger},[H, F]\right]\right| 0\right\rangle$ and $m_{3}=(1 / 2)\left\langle 0\left|\left[\left[F^{\dagger}, H\right],[H,[H, F]]\right]\right| 0\right\rangle$, and the ratio $\left(m_{3} / m_{1}\right)^{1 / 2}$ gives the desired upper bound to the energy of the states excited by the operator $F$. In order to select the surface modes, one chooses the excitation operator in the form $F=\sum_{i=1}^{N} r_{i}^{\ell} Y_{\ell \ell}\left(\theta_{i}, \phi_{i}\right)$, with $\ell \neq 0$, where $N$ is the number of atoms in the condensate. The explicit calculation of the ratio $\left(m_{3} / m_{1}\right)$ with this operator gives the dispersion law

$$
\omega^{2}(\ell)=\omega_{\perp}^{2} \ell\left[1+(\ell-1) \beta_{\ell}\right],
$$

where

$$
\beta_{\ell}=\frac{\int d \mathbf{r}\left(\nabla_{\perp} \sqrt{n}\right)^{2} r_{\perp}^{2 \ell-4}}{\int d \mathbf{r} n r_{\perp}^{2 \ell-2}}
$$

and $r_{\perp}=\left(x^{2}+y^{2}\right)^{1 / 2}$. Here $n\left(r_{\perp}, z\right)$ is the ground state density of the condensate which can be calculated by solving the stationary GP equation. The sum rule estimate is close to the exact Bogoliubov's spectrum whenever the state excited by $F$ is highly collective, with a strength which almost exhausts the dynamic structure factor. This is true for shape deformations of low multipolarity, which are the important ones for the present analysis. The dynamic structure factor associated with large $\ell$ excitations is expected to be more and more fragmented and, consequently, the sum rule upper bound becomes less accurate.

The surface state with lowest energy is the quadrupole mode. The sum rule estimate is easily obtained by inserting $\ell=2$ into Eqs. (11)-(2). One finds

$$
\omega^{2}(\ell=2)=2 \omega_{\perp}^{2}\left(1+\frac{\left(E_{\mathrm{kin}}\right)_{\perp}}{\left(E_{\mathrm{ho}}\right)_{\perp}}\right)
$$

where $\left(E_{\text {kin }}\right)_{\perp}$ and $\left(E_{\mathrm{ho}}\right)_{\perp}$ are the radial contributions to the kinetic and external potential energies, respectively. For noninteracting particles these two energies are equal and one gets $\omega=2 \omega_{\perp}$. The other interesting limit is $N \rightarrow \infty$ (Thomas-Fermi limit), when the kinetic energy in the ground state is much smaller than both the atomatom interaction energy and the trapping potential. In this limit Eq. (3) gives $\omega=\sqrt{2} \omega_{\perp}$. The latter result can also be obtained by solving the hydrodynamic equations of a superfluid 10], which provide the general dispersion law $\omega(\ell)=\sqrt{\ell} \omega_{\perp}$ for the surface modes with $m= \pm \ell$. The hydrodynamic approach, however, is accurate only for the lowest multipoles when one can completely neglect the contribution of the quantum pressure term in the GP equation; for the values of $N$ which are relevant in the current experiments, the hydrodynamic dispersion becomes soon inaccurate as $\ell$ increases. This has an important consequence for the evaluation of the critical frequency $\Omega_{c}=\min [\omega(\ell) / \ell]$, since the hydrodynamic dispersion gives $\omega(\ell) / \ell=\omega_{\perp} / \sqrt{\ell}$ and hence $\Omega_{c}=0$; conversely, $\Omega_{c}$ always exhibits a minimum, even for very large $N$, when calculated with Bogoliubov's equations or using the sum rule results (11)-(2).

In Fig. 11 we show typical results for $\omega(\ell) / \ell$, in units of $\omega_{\perp}$, obtained by inserting in Eqs. (1)-(2) the ground state density of three different condensates of ${ }^{87} \mathrm{Rb}$ atoms (we use $a=100 a_{0}$ for the scattering length, where $a_{0}$ is the Bohr radius). The stationary GP equation has been solved numerically as in Ref. 11]. The condensates have the same number of atoms, $N=10^{5}$, and the same average trapping frequency, $\omega_{\text {ho }}=\left(\omega_{x} \omega_{y} \omega_{z}\right)^{1 / 3}=\lambda^{1 / 3} \omega_{\perp}=$ $2 \pi(20 \mathrm{~Hz})$, but different asymmetry parameter: $\lambda=0.1$ (prolate), $\lambda=1$ (spherical) and $\lambda=10$ (oblate). The $N$ dependence of $\omega(\ell) / \ell$ is instead given in Fig. 2, where we plot the curves for condensates with different $N$ but the same prolate geometry: $\lambda=0.06$ and $\omega_{\text {ho }}=2 \pi(82.5 \mathrm{~Hz})$, close to the values of Ref. [6]. In order to test the accuracy of the sum rule approach, we have also solved numerically the Bogoliubov's equations for a spherical condensate. The results are shown as open circles in Fig. 11 and can be compared with the sum rule results for $\lambda=1$ (solid circles). As one can see, the accuracy of the sum rule approach is very good for the lowest values of $\ell$, remaining of the order of $10 \%$ up to $\ell=10$. This is enough for our purposes. Finally, the hydrodynamic prediction is also shown as a dashed line in both figures.

In Table I we summarize the situation for three different condensates. The parameters of the first (prolate) and the second (spherical) are close to the ones of current experiments at ENS-Paris [6] and JILA-Boulder [12], respectively. The third one (oblate) is presented in order to stress the dependence of the critical frequencies on the geometry of the trap. Actually, the critical frequencies $\omega(\ell) / \ell$ for the lowest multipoles $(\ell=2,3,4)$ turn out to be rather independent of the shape of the condensate and are well described by the hydrodynamic dispersion, which is independent of $\lambda$. For the quadrupole mode, for example, it is very well reproduced by the hydrodynamic value $\omega_{\perp} / \sqrt{2}=0.707 \omega_{\perp}$. The minimum of $\omega(\ell) / \ell$ 
instead depends on $\lambda$ in a more significant way, since it involves higher $\ell$ 's, especially for the oblate geometry.

The frequencies $\omega(\ell) / \ell$ in Table I are thresholds for the transfer of angular momentum to a condensate through shape deformations. The values of $\ell$ actually involved in a given experiment depend on the type of process used. For instance, a laser beam which makes a rotating hollow on the surface of the condensate might excite a bunch of states with several $\ell$ 's, just as happens for a hole moving through the fluid and exciting phonon-like excitations with several momentum components. Conversely, the stirred confining potential of Ref. [6], with a rotating quadrupole deformation, is expected to excite mostly quadrupole excitations. What really happens just above, or close to the threshold can not be described only in terms of elementary excitations. In fact, once a threshold is reached, the rotating perturbation can pump many excitations into the condensate, bringing it far from the linear regime. A wider configuration space is made available and the system can find new rotating equilibrium states, like the stationary deformed configurations discussed in 13, or jump into states with quantized vorticity.

Concerning vortices, an important frequency scale is fixed by the difference between the energy per particle of a condensate with and without vortex calculated in the rotating frame, $\Omega_{v}=(1 / \hbar)\left[(E / N)_{v}-(E / N)_{g}\right]$. This corresponds to the ratio between the 'excitation' energy of the vortex in the rest frame, $\left(E_{v}-E_{g}\right)$, and its angular momentum $N \hbar$. When the rotation frequency $\Omega$ is equal to $\Omega_{v}$ the energies of the configurations with and without vortex are equal in the rotating frame and, above $\Omega_{v}$, the vortex state becomes the global energy minimum. In this sense, $\Omega_{v}$ is analog to $\omega(\ell) / \ell$, since the latter represents the frequency above which a surface state of angular momentum $\ell$ has negative energy in the rotating frame [14]. A crucial difference, however, is that in order to transfer $N \hbar$ angular momentum and nucleate a vortex above $\Omega_{v}$ one has to overcome an energy barrier, while no barrier is found above $\omega(\ell) / \ell$ for the creation of quanta of angular momentum through surface excitations.

The quantity $\Omega_{v}$ can be obtained from the solution of the stationary Gross-Pitaevskii equation for the ground state and the state with a vortex line along $z$. We have already presented some numerical results for this quantity in 11]. By repeating the same kind of calculation for the three condensates in Table I we find the values given in the last column [15]. It has been noted in Ref. [6] that the experimental result for the frequency at which vortices occur is significantly larger than $\Omega_{v}$. The critical frequency in the experiment turns out to be about $0.67 \omega_{\perp}$, to be compared to the value $0.35 \omega_{\perp}$ given in the first row of Table I. This is likely to be the consequence of the above mentioned energy barrier, which prevents the nucleation of vortices even above $\Omega_{v}$. If one tries to impart a rotation to the condensate by rotating a slightly asymmetric external field, the crucial question is how angular momentum is actually transferred to the system. Our analysis suggests that, if the transfer mechanism is the creation of surface excitations of multipolarity $\ell$, the relevant threshold is $\omega(\ell) / \ell$, which can be significantly larger than $\Omega_{v}$, as shown in Table I. This argument might be used to interpret the fact that the experimental critical frequency of Ref. [6 turns out to be closer to the quadrupole value $\omega(2) / 2 \sim \omega_{\perp} / \sqrt{2}$ than to $\Omega_{v}$. Were this true, one should find almost the same critical frequency by repeating the experiment in spherical or oblate condensates. This clearly suggest that the $\lambda$-dependence could discriminate between alternative descriptions for vortex nucleation and stability (see, for instance, Refs. [16 18 and references therein). Another important signature of the suggested threshold mechanism would be the observation of strongly deformed configurations in the initial stage of the vortex nucleation. Preliminary evidence for such deformations has already emerged 19]. Finally, the threshold behavior here discussed would imply a significant hysteresis when the rotation frequency is first speeded up to produce vortices and then slowed down again, since one expects different mechanisms for the two processes: the nucleation of the vortex state at relatively high rotation frequency and the destabilization of the vortex at lower frequency (for an extensive discussion about the destabilization of vortices see [16]). The hysteresis mechanism of the vortical configurations has some similarity with the behavior of superfluid helium. In that case, however, vortex nucleation is associated with the pinning of vortex lines at the walls of the container [7]. Trapped condensates have no rough surfaces or, in other words, they have an adjustable "roughness"; in this sense, they offer a novel and complementary tool for understanding nucleation processes of quantized vorticity in superfluids.

This work has been supported by the Ministero dell'Università e della Ricerca Scientifica e Tecnologica. F.D. would like to thank the Dipartimento di Fisica di Trento for hospitality.

[1] L.D. Landau, J. Phys. (USSR) 5, 71 (1941).

[2] C. Raman et al., Phys. Rev. Lett. 83, 2502 (1999).

[3] B. Jackson, J.F. McCann, and C.S. Adams, Phys. Rev. A 61, 051603(R) (2000); T. Winiecki et al., e-print condmat/0004430.

[4] P.O. Fedichev and G.V. Shlyapnikov, e-print condmat/0004039.

[5] R. Onofrio et al., Phys. Rev. Lett. 84, 810 (2000)

[6] K.W. Madison, F. Chevy, W. Wohlleben, and J. Dalibard, Phys. Rev. Lett. 84, 806 (2000); F. Chevy, K.W. Madison and J. Dalibard, e-print cond-mat/0005221. 
[7] R.J. Donnelly, Quantized Vortices in Helium II (Cambridge University Press, Cambridge 1991). The pioneering papers by G.B. Hess and W.M. Fairbank, Phys. Rev. Lett. 19, 216 (1967) and by R.E. Packard and T.M. Sanders, Phys. Rev. A 6, 799 (1972) are also worth reading in view of the most recent analysis done in Ref. [6] on the angular momentum in trapped condensates.

[8] F. Dalfovo, S. Giorgini, L. Pitaevskii and S. Stringari, Rev. Mod. Phys. 71, 463 (1999).

[9] F. Dalfovo et al., Phys. Rev. A 56, 3840 (1997).

[10] S. Stringari, Phys. Rev. Lett. 77, 2360 (1996).

[11] F. Dalfovo and S. Stringari, Phys. Rev. A 53, 2477 (1996).

[12] M.R. Matthews et al., Phys. Rev. Lett. 83, 2498 (1999)

[13] A. Recati, F. Zambelli and S. Stringari, e-print condmat/0007152

[14] See also the discussion in T. Isoshima and K. Machida, Phys. Rev. A 60, 3313 (1999).

[15] A simple analytic expression, valid in the Thomas-Fermi limit, has been also derived by E. Lundh, C.J. Pethick and H. Smith, Phys. Rev. A 55, 2126 (1997). For the three values of $\Omega_{v}$ in Table I we checked that their approximate formula differs from the exact GP result by less than $2 \%$.

[16] A. Svidzinsky and A. Fetter, e-print cond-mat/0007139 and references therein.

[17] D.L. Feder, C.W. Clark, and B.I. Schneider, Phys. Rev. Lett. 82, 4956 (1999) and Phys. Rev. A 61, 011601 (2000); D.L. Feder et al., e-print cond-mat/0009086.

[18] J.J. García-Ripoll and V.M. Pérez-García, e-print condmat/0006368.

[19] J. Dalibard, private communication.

\begin{tabular}{||c||c|c|c|c||c||}
\hline Condensate & $\frac{\omega(2)}{2}$ & $\frac{\omega(3)}{3}$ & $\frac{\omega(4)}{4}$ & $\min \left[\frac{\omega(\ell)}{\ell}\right]$ & $\Omega_{v}$ \\
\hline \hline prolate & 0.72 & 0.61 & 0.56 & 0.53 & 0.35 \\
$\lambda=0.0058$ & & & & & \\
$\nu_{\perp}=175 \mathrm{~Hz}$ & & & & & \\
$N=2.5 \times 10^{5}$ & & & & & \\
\hline spherical & 0.71 & 0.59 & 0.53 & 0.44 & 0.24 \\
$\lambda=1$ & $(0.71)$ & $(0.59)$ & $(0.52)$ & $(0.41)$ & \\
$\nu_{\perp}=7.8 \mathrm{~Hz}$ & & & & & \\
$N=3 \times 10^{5}$ & & & & & \\
\hline oblate & 0.71 & 0.58 & 0.50 & 0.33 & 0.12 \\
$\lambda=10$ & & & & & \\
$\nu_{\perp}=10 \mathrm{~Hz}$ & & & & & \\
$N=3 \times 10^{5}$ & & & & & \\
\hline \hline
\end{tabular}

Table I. Critical frequencies for shape deformations in different condensates. First column: type of condensate. Next four columns: critical frequency $\omega(\ell) / \ell$, in units of the radial trapping frequency $\omega_{\perp}=2 \pi \nu_{\perp}$, obtained with the sum rule expressions (11)-(2) for $\ell=2,3,4$ and for its minimum value. The numbers in brackets are the exact results of Bogoliubov's equations in the spherical case. The last column is the quantity $\Omega_{v}=(1 / \hbar)\left[(E / N)_{v}-\right.$ $\left.(E / N)_{g}\right]$ (see text).

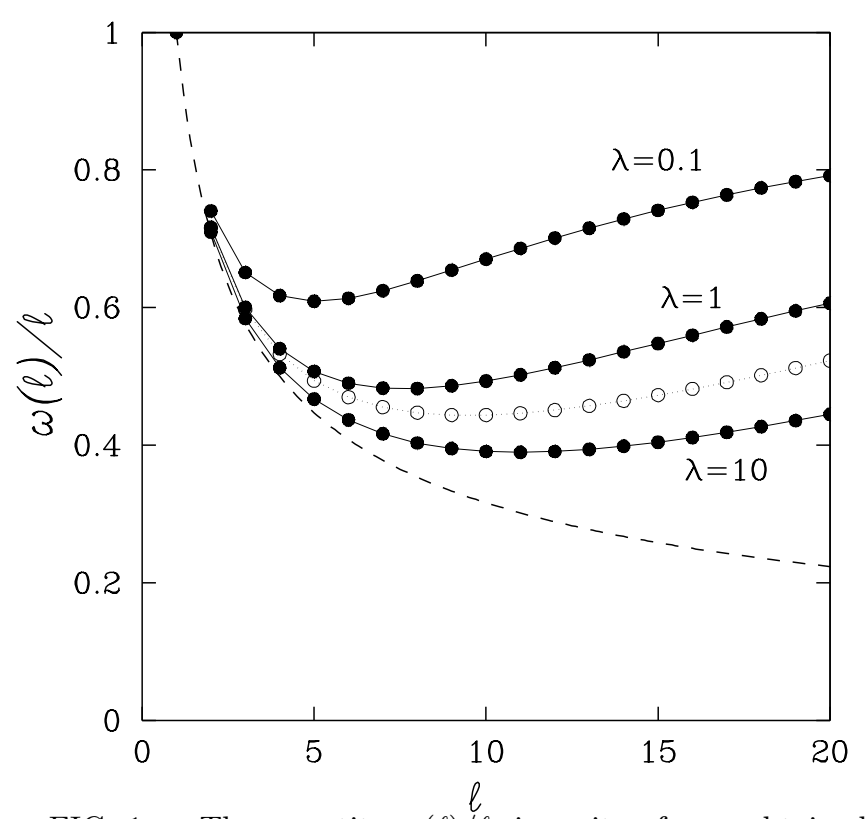

FIG. 1. The quantity $\omega(\ell) / \ell$, in units of $\omega_{\perp}$, obtained with Eqs. (1)-(2) as a function of $\ell$ for three condensates of ${ }^{87} \mathrm{Rb}$ atoms with different values of $\lambda=\omega_{z} / \omega_{\perp}$ : prolate $(\lambda=0.1)$, spherical $(\lambda=1)$, oblate $(\lambda=10)$. The three condensates have the same value of $N=10^{5}$ and $\omega_{\text {ho }}=\lambda^{1 / 3} \omega_{\perp}=2 \pi(20 \mathrm{~Hz})$. Open circles correspond to the exact solution of the Bogoliubov's equation in the spherical case $(\lambda=1)$. The dashed line is the hydrodynamic prediction $\omega(\ell) / \ell=1 / \sqrt{\ell}$.

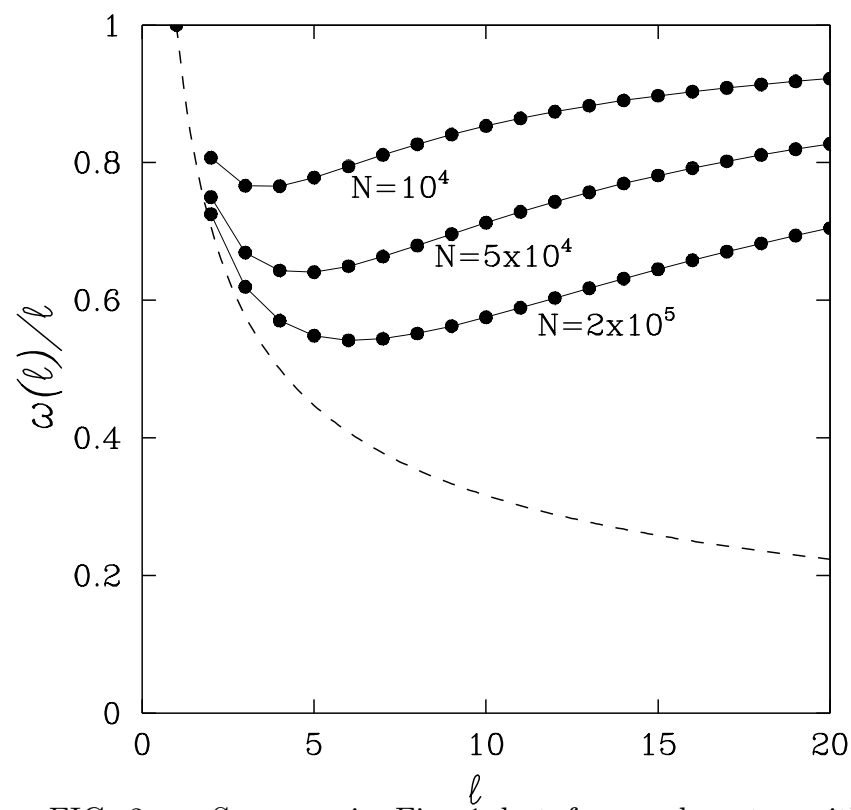

FIG. 2. Same as in Fig. 1 but for condensates with different values of $N$. All condensates have the same $\omega_{\text {ho }}=2 \pi(82.5 \mathrm{~Hz})$ and $\lambda=0.06$. The parameters used for the curve $N=2 \times 10^{5}$ are close to the actual situation in the experiments of Ref. [6]. 